\title{
Measurement error in the Hawksley random zero sphygmomanometer: what damage has been done and what can we learn?
}

\author{
Rónán M Conroy, Eoin O’Brien, Kevin O’Malley, Neil Atkins
}

\begin{abstract}
The Hawksley random zero sphygmomanometer is used in all aspects of blood pressure research, from clinical trials to evaluation of new blood pressure recorders. It is designed to reduce observer bias in blood pressure measurement. The problem is that it also underestimates blood pressure. Furthermore, this was first reported more than two decades ago. In this paper Rónán Conroy and colleagues explore the consequences of using an inaccurate instrument for important research and why prestigious organisations like the World Health Organisation continue to use it.
\end{abstract}

But if the salt have lost his savour, wherewith shall it be salted?

MATTHEW v, 13.

We recently reported that the Hawksley random zero sphygmomanometer underestimated both systolic and diastolic blood pressure when compared with a mercury sphygmomanometer. Following the protocol of the British Hypertension Society, using large numbers of measurements with interchanged observers, we found that when measuring systolic pressure the Hawksley instrument read on average $3.5 \mathrm{~mm} \mathrm{Hg}$ lower than the mercury instrument $(95 \%$ confidence interval 3.4 to $4.2 \mathrm{~mm} \mathrm{Hg}$ ) and that when measuring diastolic pressure it read $7.5 \mathrm{~mm} \mathrm{Hg}$ lower $(7.0$ to 8.0 $\mathrm{mm} \mathrm{Hg}$ ). These differences have serious implications for research results based on data gathered with the Hawksley instrument. What is more serious, however, is that doubts about the Hawksley random zero sphygmomanometer had been voiced as long ago as 1970 , so that a large body of published research has subsequently accumulated whose interpretation may be in doubt. ${ }^{1}$

\section{Brief history of Hawksley random zero instrument}

In 1963 Garrow described a "zero-muddler for unprejudiced sphygmomanometry," 2 which was subsequently developed ${ }^{3}$ and marketed as the Hawksley random zero sphygmomanometer (Hawksley and Sons, Lancing, Sussex). The essential principle of the instrument is that it blinds the observer to the actual blood pressure until after the reading has been made. It does this by adding a random amount of mercury to the manometer as the reading is being taken. When the instrument falls back to zero the amount of extra mercury can be read and subtracted. The design is simple and ingenious. It really ought to work.

In 1970, almost as soon as the random zero sphygmomanometer became available, Evans and Prior reported that it read slightly but significantly lower than a mercury sphygmomanometer. ${ }^{4}$ This problem was also noted in 1973 in a paper reviewing the comparative performance of several blood pressure measuring devices. ${ }^{5}$ No concern seems to have been raised by these findings, however, and in the course of time the Hawksley instrument became popular in epidemiological research, favoured by such prestigious institutions as the Medical Research Council and the World Health Organisation.
In 1985 Gaudemaris et al reported a significant correlation between the zero level used by the Hawksley sphygmomanometer and the difference between the resulting blood pressure and the blood pressure obtained with a standard mercury instrument. ${ }^{6}$ In the same year Silman showed that although the Hawksley instrument reduced terminal digit preference, it did not eliminate it as the originators had claimed. ${ }^{7}$ These results were quickly corroborated by Hosie and Hosie. ${ }^{8}$ Three years later Parker et al published data suggesting that there was a serious problem with accuracy, ${ }^{9}$ and in 1990 a group of prominent American researchers concluded that the "random zero instrument does not function the way it is understood to" and called for a formal study of the Hawksley device. ${ }^{10}$

That formal evaluation, carried out with both currently accepted protocols for evaluating blood pressure measuring devices, was published by us in 1990. It showed that the concerns expressed by other workers were amply justified: the Hawksley random zero sphygmomanometer systematically underestimates blood pressure.' The study protocol was the most stringent evaluation to which the instrument had been subjected. The study was run with two machines -one British model, one American-selected from three of each type supplied by the manufacturers, who knew that they were required for formal evaluation. The machines had previously been compared to verify that they did not differ among themselves. As it was most unlikely that the manufacturers unwittingly supplied six similarly defective machines our findings must be taken as evidence of a generic fault.

Despite the accumulation of such comprehensive evidence against the Hawksley instrument, there is little sign that its popularity is waning. Two important questions arise: what are the consequences of the measurement error of the instrument for interpreting the findings of the many studies which have used it? and why has the instrument continued in use, unmodified, in the face of a growing and now definitive body of evidence that it is inaccurate?

\section{Implications for published research}

The most common uses of the Hawksley sphygmomanometer are in research directed at improving blood pressure recording protocols, in community surveys and other epidemiological studies, in defining patient populations for treatment trials in hypertension, and in assessing the performance of automated blood pressure measuring devices.

IMPROVING BLOOD PRESSURE RECORDING PROTOCOLS

In studies aimed at improving protocols for blood pressure measurement use of the Hawksley sphygmomanometer is unlikely to have adverse effects. Typical studies in which the instrument was used include one by Whincup et al, which examined the effect of cuff size on blood pressure measurement in children ${ }^{11}$; one by Canner et al, which examined the sources of variability in clinical blood pressure readings ${ }^{12}$; and

Dr Rónán Conroy,

Epidemiology. 


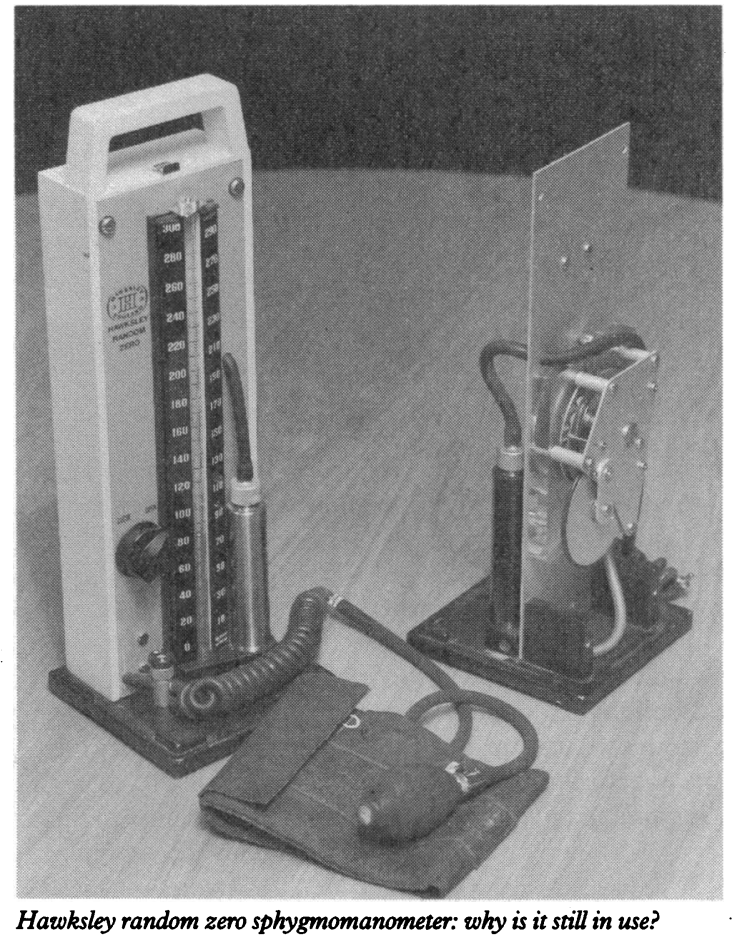

one by van Loo et al, which examined the effect on the prevalence of isolated systolic hypertension of letting subjects rest for 25 minutes before measuring blood pressure. ${ }^{13}$ In these studies, although blood pressure readings (and, in the last case, hypertension levels) would have erred on the low side, the study data were analysed by comparing one set of Hawksley readings with another, so that the conclusions reached would not have been affected by measurement bias.

\section{COMMUNITY AND OTHER EPIDEMIOLOGICAL STUDIES}

It is ironic that community surveys that have tried to quantify blood pressure more precisely have used an unvalidated measuring instrument and thereby produced descriptive statistics which are biased. The WHO's CINDI project, which is an international initiative fostering countrywide integrated noncommunicable diseases intervention programmes, specifies use of the Hawksley sphygmomanometer in its protocol. ${ }^{14}$ The instrument is likewise specified in the protocol of the WHO's MONICA project, which is monitoring cardiovascular risk factors and events in countries around the world. ${ }^{15}$ The MONICA project is one of the largest systematic exercises in measuring blood pressure at community level ever undertaken. Now the results of the entire project will have to be corrected for measurement bias.

The situation is compounded by the fact that, although the MONICA protocol specifies a Hawksley sphygmomanometer, some countries that have not the funds to equip survey teams with Hawksley instruments are instead using mercury sphygmomanometers. Though the data gathered may be used to calculate the relation between increasing blood pressure and increasing cardiovascular risk within communities, the absolute blood pressure readings and the estimated prevalence of hypertension will be biased downwards. Hence the data will have to be adjusted before results from centres using the Hawksley instrument can be pooled with those from countries using mercury instruments.

Many other community studies also routinely use the Hawksley sphygmomanometer. Barnowski et al studied ethnic variation in blood pressure among preadolescent children. ${ }^{16}$ The associations found remain valid but the absolute values were biased. In Ireland the Kilkenny health project, in common with many community programmes for the prevention of cardiovascular disease in other countries, has used the Hawksley instrument for its pre- and post-programme surveys. ${ }^{17}$ As these programmes aim at reducing cardiovascular risk factors, using the Hawksley random zero device will not mask a change in blood pressure. Nevertheless, the absolute levels of blood pressure reported will be biased as, in addition to their value as experiments, these programmes also provide valuable data on levels of risk factors in representative samples of communities.

One area in which use of the Hawksley will have contributed a legacy of confusion is in projects that pooled data collected with Hawksley and conventional instruments. The valuable report by de Man et al, ${ }^{18}$ which pooled data from six population based studies producing a total database of 28043 children, unfortunately combined data from three studies which used a Hawksley and three which used mercury sphygmomanometers. The situation is compounded by the fact that two of the remaining three studies used the London School of Hygiene sphygmomanometer, which has also been shown to be inaccurate and to disagree with the Hawksley. ${ }^{19}$ There is thus no simple way of correcting the invaluable centile reference charts which the authors published showing blood pressure levels by age.

A related problem occurs in the use of large scale follow up studies to generate functions for estimating the risk burden of individuals. These functions have been used in counselling individuals, notably by worksite health programmes, to give a composite index of cardiovascular risk to screenees. Unfortunately, basing such a calculation on a logistic function derived from any study which used the Hawksley will result in an overestimate of the risk to an individual-especially if the function uses diastolic blood pressure. There is considerable variation in the weighting that functions derived from different studies attach to blood pressure. A $1 \mathrm{~mm} \mathrm{Hg}$ rise in systolic pressure, for example, would lead to a $0.6 \%$ increase in risk in the function published by Keyes $e t$ al from data on middle aged men collected in the twin cities study, ${ }^{20}$ but other groups have reported an increase of $0.24 \%$ to $0.33 \%$ in relative risk. ${ }^{21-23}$ The use of a conventional mercury instrument rather than a Hawksley in measuring the blood pressure of an individual would therefore result.in only a very small change in absolute risk, so it is unlikely that people are being seriously misinformed as a result of inaccuracies in logistic functions derived from blood pressure measured by the Hawksley.

DEFINING PATIENT POPULATIONS FOR TREATMENT TRIALS

The use of the Hawksley instrument for selecting patients to be included in treatment trials of antihypertensive agents has more serious consequences. Such trials generally recruit patients based on a minimum blood pressure level, such as the trial reported by Verdecchia et al, ${ }^{24}$ who compared duration of effect of two antihypertensive drugs. They used the Hawksley to recruit patients whose diastolic pressure was greater than $100 \mathrm{~mm} \mathrm{Hg}$. Other trials using the Hawksley, such as that reported by Kane et al have used a cut off point of $90 \mathrm{~mm} \mathrm{Hg}$. While the end points studied appear uninfluenced by the bias inherent in the use of the Hawksley, as they involved assessing and comparing changes in blood pressure, the use of the Hawksley introduces a selection bias of unknown effect. These studies were not, as reported, on patients with diastolic pressures over 100 and $90 \mathrm{~mm} \mathrm{Hg}$, respectively, but on patients with pressures over 107.5 and $97.5 \mathrm{~mm} \mathrm{Hg}$.

As the treatment of mild hypertension, characterised by diastolic pressures of between 90 and 100 $\mathrm{mm} \mathrm{Hg}$ continues to be the subject of debate ${ }^{25}$ it is 
unfortunate that several of the large scale studies designed to clarify the value of treatment in this group have been conducted on patients recruited on the basis of Hawksley sphygmomanometer blood pressure readings. We must face the prospect that the knowledge we thought we had acquired about the treatment of patients with diastolic pressures between 90 and 100 $\mathrm{mm} \mathrm{Hg}$ actually relates to those with pressures between $97 \cdot 7$ and $107.5 \mathrm{~mm} \mathrm{Hg}$.

The cost of this loss of data is incalculable and some results may need extensive reanalysis. For example, the Australian therapeutic trial in mild hypertension used the Hawksley random zero sphygmomanometer and the London School of Hygiene sphygmomanometer interchangeably. ${ }^{26}$ Not only is there the likelihood, therefore, that studies designed to assess treatment in patients in the $90-100 \mathrm{~mm} \mathrm{Hg}$ diastolic range wasted time and money by including the wrong patients but what we know of the benefits of treatment in this group may also be wrong. Guidelines for the treatment of mild hypertension may have been formulated on the basis of data gathered from people with moderate hypertension.

The primary prevention with metoprolol in patients with hypertension (MAPHY) study, ${ }^{27}$ for example, included 1209 patients over 8110 patient years of follow up. Patients were selected on the basis of diastolic pressure readings taken with a Hawksley random zero sphygmomanometer. Those included in the trial had diastolic pressures of $100-129 \mathrm{~mm} \mathrm{Hg}$ but, had they been assessed with a standard sphygmomanometer, would have been in the range $107 \cdot 5-136 \cdot 5$ $\mathrm{mm} \mathrm{Hg}$-clearly a more hypertensive group than intended.

The task of reviewing the methodology of large scale treatment trials in hypertension for selection bias associated with the use of the Hawksley instrument is one which bodies responsible for setting treatment guidelines should undertake without delay.

\section{ASSESSING PERFORMANCE OF AUTOMATED DEVICES}

The final and a commonly reported use of the Hawksley is in assessing the accuracy of automated blood pressure measuring devices. The Hawksley is used as the standard against which other techniques are assessed. Not surprisingly, given what we know now about the performance of the Hawksley, many such studies reported favourably on the automated instrument but criticised it for overestimating blood pressure. Rogers et al, for example, found that the Copal UA-231 read $2.3 \mathrm{~mm} \mathrm{Hg}$ higher than the Hawksley for systolic and $1.9 \mathrm{~mm} \mathrm{Hg}$ higher for diastolic pressure. ${ }^{28}$ Overestimation of systolic pressure was reported by Maheswaran et al, who studied the Copal UA-251 and Dinamap 1848 machines. ${ }^{29}$ Fouqueray et al ${ }^{30}$ who also studied agreement between the UA-251 and the Hawksley, found both systolic and diastolic pressures were higher when measured by the electronic instrument. Whincup et al, comparing a Dinamap 1846SX instrument with the Hawksley, reported that the Dinamap overestimated systolic pressure by a mean of $8.1 \mathrm{~mm} \mathrm{Hg}^{31}$ They also reported that differences between the two machines were related to blood pressure level, an effect which may be due to the relation of the inaccuracy of the Hawksley instrument to blood pressure level, and may not reflect a genuine inaccuracy in the Dinamap 1846SX.

A study reported by Ornstein et al is especially significant. They found that the Dinamap automated blood pressure measuring device seriously overestimated blood pressure readings when compared with the Hawksley and concluded, "The results of this study would suggest that routine use of the Dinamap 8100 would lead to serious misclassification errors in screening for hypertension and in the follow-up of known hypertensive patients." ${ }^{32}$ We do not know, on the basis of the data reported in the study, whether the Dinamap 8100 does, indeed, have a high misclassification rate; the results could also have been a result of the use of the Hawksley. To assess the consequences of using a Hawksley as a standard against which to assess automated instruments we reanalysed data gathered during the validation of the SpaceLabs $90202 . .^{33}$ We concluded that, validated against a Hawksley, the SpaceLabs 90202 would have achieved a British Hypertension Association grade of D for both systolic and diastolic pressure measurements - the lowest grade possible. In fact, assessed against a mercury instrument it achieved grades of $\mathrm{C}$ for systolic and B for diastolic pressure measurements (unpublished data). The potential damage done to commercial interests by using an inaccurate device as a standard should not be underestimated.

Automated blood pressure measurement is a growing and competitive science, and the use of the Hawksley as a standard is paradoxically giving an advantage to instruments whose bias coincides with that of the Hawksley. What we do not know is the extent to which manufacturers are using the Hawksley in the development and calibration of automated blood pressure recording devices, leading to machines which will perform well in comparison with the Hawksley but which perpetuate the errors of the Hawksley. Not only it is not possible to estimate the cost of the studies whose results have been thrown into doubt by the finding that their standard was inappropriate but, in addition, the costs of these studies to manufacturers of what may well be excellent blood pressure measuring devices may only be guessed at.

\section{Why is the Hawksley still in use?}

When will we learn to extend the reasonable doubt that we have of the objectivity of the human observer to the accuracy of the instruments we use? It is remarkable that an instrument so widely used in the measurement of blood pressure was not itself subjected to assessment. If the loss of valuable data which we have described had happened as a result of a problem that no one knew about it would be tragic. But reports questioning the accuracy of the Hawksley data from 1970 , and the accumulated evidence is now such that the use of the Hawksley as a blood pressure measuring instrument cannot be justified. Perhaps the vast body of data collected with use of the Hawksley is a deterrent to change. There is also a psychological resistance to believing that something which looks as if it should work is, in fact, faulty. And there is also something reassuring about consensus-"what's good enough for the WHO is good enough for me."

Nevertheless, it verges on scandalous that the accumulating evidence has been ignored for two decades and vast sums in scarce medical research resources have been spent gathering data with an inaccurate instrument. And there is no sign that the popularity of the Hawksley is waning. Research protocols pass through the hands of many peopleethics committees, funding bodies, journal referees, and editors - and it is sadly apparent that nowhere in this chain of review is there anyone with the initiative to say stop. Where is John Snow now that we need him?

1 O'Brien E, Mee F, Atkins N, O'Malley K. Inaccuracy of the Hawksley random zero sphygmomanometer. Lancet 1990;336:1465-8.

2 Garrow JS. Zero-muddler for unprejudiced sphygmomanometry. Lance 1963;iv: 1205.

3 Wright BM, Dore CF. A random zero sphygmomanometer. Lancet 1970; 337-8.

4 Evans JG, Prior IAM. Experience with the random-zero sphygmomanometer Br J Prev Soc Med 1970;24:10-5.

5 Labarthe DR, Hawkins CM, Remington RD. Evaluation of performance of 
selected devices for measuring blood pressure. Am $\mathcal{f}$ Cardiol 1973;32: 546-53.

6 Gaudemaris RD, Folsom AR, Prineas RJ, Luepker RV. The random-zero versus the standard mercury sphygmomanometer: a systematic bloodpressure difference. Am F Epidemiol 1985;121:282-90.

7 Silman AJ. Failure of random zero sphygmomanometer in general practice. $B M F$ 1985;290:1781-2.

8 Hosie GAC, Hosie J. "Failure" of the random zero sphygmomanometer in general practice. $B M \mathcal{F}^{1}$ 1985;291:137-8.

9 Parker D, Liu K, Dyer AR, Giumetti D, Liao Y, Stamler J. A comparison of the random-zero and standard mercury sphygmomanometers. Hypertension $1988 ; 1$

10 Kronmal RA, Rutan GH, Borhani NO, Manolio TA, Furberg CD. Potentia problems with random zero sphygmomanometer. Lancet 1990;335:360.

11 Whincup PH, Cook DG, Shaper AG. Blood pressure measurement in children: the importance of cuff bladder size. $f$ Hypertens 1989;7:845-50.

12 Canner PL, Borhani NO, Oberman A, Cutler J, Prineas RJ, Langford H, Hooper FJ. The hypertension prevention trial: assessment of quality of blood pressure measurements. Am $\mathcal{F}$ Epidemiol 1991;134:379-92.

13 van Loo JM, Peer PG, Thien TA. Twenty-five minutes between blood pressure readings: the influence on prevalence rates of isolated systolic hypertension. F Hyperens 1986;4:631-5.

14 Leparski E, Nüssel N, eds. CINDI. Countrywide integrated noncommunicable diseases intervention programme. In: Protocol and guidelines for monitoring and evaluation procedures. Berlin: Springer Verlag, 1987:40-2.

15 WHO MONICA Project Principal Investigators. The World Health Organization MONICA project (monitoring trends and determinants in cardiovascular disease): a major international collaboration. 7 Clin Epidemiol 1988;41:105-14

16 Barnowski T, Tsong Y, Henske J, Dunn JK, Hooks P. Ethnic variation in blood pressure among preadolescent children. Pediatr Res 1988;23:270-4

17 Shelley E, Drynan J, Conroy R, Cuddihy J, Lee B, Magnier P. Risk factors for coronary heart disease: a population survey in County Kilkenny, Ireland, in 1985. Ir J Med Sci 1991;160(suppl 9):22-8.

18 de Man SA, André J-L, Bachmann H, Grobbee DE, Ibsen KK, Laaser U, et al. Blood pressure in childhood: pooled findings of six European studies. f Hypertens 1991;9:109-14.

19 Fitzgerald DJ, O'Malley K, O'Brien ET. Inaccuracy of the London School of Hygiene sphygmomanometer. $B M \mathcal{F}^{1} 1982 ; 284: 18-9$.

20 Keys A, Longstreet Taylor H, Blackburn H, Brozek J, Anderson JT, Simonson E. Mortality and coronary heart disease among men studied for 23 years. Arch Intern Med 1971;128:201-14.
21 Keys A, Karvonen MJ, Punsar S, Menotti A, Fidanza F, Farchi G. HDL serum cholesterol and 24-year mortality of men in Finland. Int $f$ Epidemiol 1984;13:428-35.

22 Keys A, Menotti A, Aravanis C, Blackburn H, Djordevic BS, Buzina R, et al. The seven countries study: 2289 deaths in 15 years. Prev Med 1984;13: $141-54$.

23 Italian Research Group of the Seven Countries Study. Twenty-five year incidence and prediction of coronary heart disease in two Italian rural population samples. Acta Cardiol 1986;4:283-99.

24 Verdecchia P, Gatteschi C, Benemio G, Boldrini F, Guerrieri M, Porcellati C. Duration of the antihypertensive action of atenolol, enalapril and placebo: a randomised within-patient study using ambulatory blood pressure. Int f Clin Pharmacol Ther Toxicol 1988;26:570-4.

25 Oglesby P. The Medical Research Council trial. Hypertension 1986;8:733-6.

26 ATT Management Committee of the Australian Therapeutic Trial in Mild Hypertension. The Australian therapeutic trial in mild hypertension. Lancet 1980;i:1261-7.

27 Wikstrand J, Warnold I, Olsson G, Tuomilehto J, Elmfeldt D, Berglund G. Primary prevention with metoprolol in patients with hypertension. $9 A M A$ 1989;259:1976-82.

28 Rogers S, Smith GD, Doyle W. Field evaluation of the Copal UA-231 automatic sphygmomanometer. $f$ Epidemiol Community Health 1948;42. $321-4$

29 Maheswaran R, Zezulka AV, Gill JS, Beevers M, Davies P, Beevers DG. Clinical evaluation of the Copal UA-251 and the Dinamap 1848 automatic blood pressure monitors. I Med Eng Technol 1988;12:160-3.

30 Fouqueray B, Julien J, Pagny JY, Jeunemaitre X, Sassano P, Battaglia C. Validation of a self-measurement blood pressure device. Arch Mal Coeur 1988;81:231-4. (In French.)

31 Whincup PH, Bruce NG, Cook DG, Shaper AG. The Dinamap 1846SX automated blood pressure recorder: comparison with the Hawksley random zero sphygmomanometer under field conditions. I Epidemiol Community Health 1992;46:164-9.

32 Ornstein S, Markert G, Litchfield L, Zemp L. Evaluation of the Dinamap blood pressure monitor in an ambulatory primary care setting. 9 Fam Pract 1988;26:517-21.

33 O'Brien E, Mee F, Atkins N, O'Malley K. Evaluation of the SpaceLabs 90202 non-invasive ambulatory recorder according to the AAMI standard and BHS criteria. 7 Hum Hypertens 1991;5:223-6.

(Accepted 16 February 1993)

\title{
How to Do It
}

\section{Teaching communication skills to clinical students}

\author{
I C McManus, C A Vincent, S Thom, J Kidd
}

\begin{abstract}
Seven years' experience in teaching communication skills to first year clinical students at St Mary's Hospital School of Medicine is described. The first component consists of a day during the introductory clinical course; this is divided into a lecture and small seminar groups and involves behavioural scientists and clinicians from many departments. The second component uses simulated patients and video feedback and takes place in small groups later in the year. Participation of the students through active critical discussion, role play, and interactive video feedback are important aspects in the success of the course. The methods have been refined through evaluation by students and tutors. This article aims to allow others, already running or considering such a course, to develop effective courses within the practical constraints of their own institutions.
\end{abstract}

St Mary's Hospital Medical School, Imperial College of Science, Technology, and Medicine, London W2 1PG I C McManus, senior lecturer in psychology

C A Vincent, lecturer in psychology

S Thom, senior lecturer in

clinical pharmacology

$\mathrm{J}$ Kidd, lecturer in

communication skills

Correspondence to:

Dr McManus.

$B M 7$ 1993;306:1322-7 positively influences health ${ }^{23}$ Poor communication has been implicated in medical accidents ${ }^{4}$ and in subsequent litigation. ${ }^{5}$ Complaints about doctors by the public usually do not deal with clinical competence but with problems of communication. ${ }^{6}$
Teaching of communication seems to be both feasible and effective. Although some early research findings have been criticised, ${ }^{7}$ other studies have clearly shown beneficial outcomes. ${ }^{36-12}$ Without specific training, medical students' communication skills seem to decline during medical training. ${ }^{10} 1113-15$

Teaching of communication skills has been slow to develop. In 1983 Wakeford found that one third of British medical schools offered no communication skills teaching, and training in the others typically amounted to only one or two hours of video recording and replay. ${ }^{16}$ By the time of the 1992 GMC survey there was some improvement; only three out of 28 medical schools did not teach any communication skills, although it was usually taught as part of general practice or psychiatry, with only four schools including teaching within general medicine and surgery. ${ }^{17}$ In 1991 Whitehouse reported that teaching of communication skills accounted for less than $2 \%$ of curriculum time.$^{18}$ Methods varied from formal lectures to analysis of student interviews. Video feedback was a part of only some courses, and students seldom had the opportunity to experience more than one or two sessions of feedback. Role play was commonly used in group work but the use of simulated patients was rare. Assessment was usually subjective and given only by the doctor or tutor. The GMC survey found that only seven schools included communication skills as a formal part of final examinations. ${ }^{17}$ In 1992 the University of London stated that newly qualified doctors should be competent in communication skills and have received formal training in the subject. ${ }^{19}$

St Mary's Hospital Medical School has provided general communication skills training to first year 